\title{
Deep inelastic prompt photon production at HERA in the $k_{T}$-factorization approach
}

\author{
Nikolay Zotov* ${ }^{\dagger}$ \\ SINP, Lomonosov Moscow State University \\ E-mail: zotovetheory.sinp.msu.ru
}

\begin{abstract}
.We investigate the prompt photon production in deep inelastic scattering at HERA in the framework of $k_{T}$-factorization QCD approach. Our study is based on the off-shell partonic process $e q^{*} \rightarrow e^{\prime} \gamma q$, where the photon radiation from the leptons as well as from the quarks is taken into account. The unintegrated quark densities in a proton are determined using the Kimber-MartinRyskin prescription. We investigate both inclusive and jet associated prompt photon production rates. Our predictions are compared with the recent experimental data taken by the H1 and ZEUS collaborations.
\end{abstract}

XVIII International Workshop on Deep-Inelastic Scattering and Related Subjects, DIS 2010 April 19-23, 2010

Firenze, Italy

\footnotetext{
${ }^{*}$ Speaker.

${ }^{\dagger}$ In collaboration with S.P. Baranov (Lebedev Physics Institute, Moscow) and A.V. Lipatov (SINP, Lomonosov Moscow State University).
} 


\section{Itroduction}

Studying the photon production in deep inelastic scattering (DIS) provides a test of perturbative Quantum Chromodynamics (QCD) with two hard scales: $E_{T}^{\gamma}$, the transverse energy of the emitted photon, and $Q^{2}$, the exchanged photon virtuality. Good understanding of the production dynamics is also important for searches of new particles decaying to photons at the LHC conditions.

At the leading-order of QCD, the production of the prompt photons in deep inelastic ep scattering is described by the parton-level process $e q(\bar{q}) \rightarrow e^{\prime} \gamma q(\bar{q})$. The observed final-state photon can be emitted by a quark or by a lepton (the so-called QQ and LL mechanisms, respectively). The interference contribution (LQ) is expected to be small. The QQ mechanism includes the direct photon radiation from the quark line and also the so-called fragmentation processes, where the produced quark forms a jet containing a photon with large fraction of the jet energy. However, the isolation criterion introduced in experimental analyses substantially reduces the fragmentation component.

Recently the H1 and ZEUS collaborations have reported their data [1, 2, 3] on the deep inelastic production of prompt photon at HERA, both inclusive and in association with a hadronic jet. A substantial (by a factor of about 2) disagreement between the data and the results of the LO pQCD $\mathscr{O}\left(\alpha^{3}\right)$ calculations [4] has been found [3] at low $Q^{2}$ (for more details see [5]).

In the present study instead of using the standard (collinear) approximation we apply the $k_{T^{-}}$ factorization approach. We have applied this approach to the inclusive and jet associated prompt photon photo- and hadroproduction at HERA [6] and the Tevatron [7]. As it was demonstrated in the ZEUS paper [8] and also in the recent study [9] performed by the H1 collaboration, the $k_{T}$-factorization predictions are in better agreement with the data than the results of collinear NLO calculations. We have extended our investigations [6] to the DIS region [10] and present here certain of these results shortly.

\section{Theoretical framework}

Let us start with the LL production mechanism. There are two simple Feynman diagrams which describe this partonic subprocess at the leading order in $\alpha_{\mathrm{em}}$. The relevant matrix element reads

$$
\mathscr{M}_{L L}\left(e q^{*} \rightarrow e^{\prime} \gamma q\right)=e_{q} e^{3} \varepsilon^{\mu} \frac{1}{q^{2}} L^{\mu v} H^{v},
$$

where leptonic and hadronic tensors are given by the following expressions:

$$
\begin{gathered}
L^{\mu v}=\bar{u}\left(p_{e^{\prime}}\right)\left[\gamma^{\mu} \frac{\hat{p}_{e^{\prime}}+\hat{p}_{\gamma}+m_{e}}{\left(p_{e^{\prime}}+p_{\gamma}\right)^{2}-m_{e}^{2}} \gamma^{v}+\gamma^{v} \frac{\hat{p}_{e}-\hat{p}_{\gamma}+m_{e}}{\left(p_{e}-p_{\gamma}\right)^{2}-m_{e}^{2}} \gamma^{\mu}\right] u\left(p_{e}\right), \\
H^{v}=\bar{u}\left(p_{q}\right) \gamma^{v} u(k) .
\end{gathered}
$$

The off-shell quark spin density matrix was taken in the form [10] $u \bar{u}=x \hat{p}$ with $p$ and being the initial proton momentum and $k=x p+k_{T}$ the quark momentum. Our expressions for the matrix elements have continuous on-shell limit, and so, can safely be used in all regions of $x$. 



Figure 1: Differential cross sections of the inclusive deep inelastic prompt photon production as a function of $E_{T}^{\gamma}, \eta^{\gamma}$ and $Q^{2}$ calculated at $3<E_{T}^{\gamma}<10 \mathrm{GeV},-1.2<\eta^{\gamma}<1.8,4<Q^{2}<150 \mathrm{GeV}^{2}, E_{e}^{\prime}>10 \mathrm{GeV}$, $153^{\circ}<\theta_{e}^{\prime}<177^{\circ}, y>0.05$ and $W_{X}>50 \mathrm{GeV}$. The solid histogram corresponds to the default scale $\mu^{2}=Q^{2}$, whereas the the upper and lower dashed histograms correspond to scale variations. The experimental data are from $\mathrm{H} 1$ [3].

The expression for $\mathscr{M}_{Q Q}\left(e q^{*} \rightarrow e^{\prime} \gamma q\right)$ can be obtained from (1) - (3) if we replace $p_{e} \rightarrow k$, $p_{e^{\prime}} \rightarrow p_{q}, k \rightarrow p_{e}, p_{q} \rightarrow p_{e^{\prime}}$ and multiply (1) by an extra factor $e_{q}$ (see Appendix in [10]).

According to the $k_{T}$-factorization theorem, the cross section of the process $e p \rightarrow e^{\prime} \gamma X$ can be written as a convolution of the off-shell matrix element squared and averaged over initial gluon polarizations and colors $\left|\overline{\mathscr{M}}\left(e q^{*} \rightarrow e^{\prime} \gamma q\right)\right|^{2}$ and unintegrated quark distribution $f_{q}\left(x, \mathbf{k}_{T}^{2}, \mu^{2}\right)$ : We have used the unintegrated quark densities in a proton $f_{q}\left(x, \mathbf{k}_{T}^{2}, \mu^{2}\right)$ which are taken in the KMR form [11]. The KMR approach is a formalism to construct the unintegrated parton (quark and gluon) distributions from the known conventional parton distributions $x a\left(x, \mu^{2}\right)$, where $a=g$ or $a=q$. For the input, we have used the recent leading-order MSTW [12] parton densities.

We choose the renormalization and factorization scales to be $\mu^{2}=\xi Q^{2}$. In order to estimate the theoretical uncertainties of our calculations we vary the scale parameter $\xi$ between $1 / 2$ and 2 about the default value $\xi=1$. We use the LO formula for the strong coupling constant $\alpha_{s}\left(\mu^{2}\right)$ with $n_{f}=4$ active (massless) quark flavours and $\Lambda_{\mathrm{QCD}}=200 \mathrm{MeV}$, such that $\alpha_{s}\left(M_{Z}^{2}\right)=0.1232$.

According to the QCD factorization the calculated cross section can be split into two pieces

$$
d \sigma=d \sigma_{\text {direct }}\left(\hat{\mu}^{2}\right)+d \sigma_{\text {fragm }}\left(\hat{\mu}^{2}\right)
$$

with $d \sigma_{\text {direct }}\left(\hat{\mu}^{2}\right)$ representing the perturbative contribution and $d \sigma_{\text {fragm }}\left(\hat{\mu}^{2}\right)$ the fragmentation contribution. In our calculations we choose the fragmentation scale $\hat{\mu}^{2}$ to be the invariant mass of the quark + photon subsystem, $\hat{\mu}^{2}=\left(p+p_{i}\right)^{2}$, and restrict $d \sigma_{\text {direct }}\left(\hat{\mu}^{2}\right)$ to $\hat{\mu} \geq M \simeq 1 \mathrm{GeV}$. Under this condition, the contribution $d \sigma_{\text {direct }}\left(\hat{\mu}^{2}\right)$ is free from divergences. We have checked that thesensitivity of our results to the choice of $M$ is reasonably soft. As far as the fragmentation contribution is concerned, its size is dramatically reduced by the photon isolation cuts (see below). Concerning the isolation cut it is the following. As in the experimental analyses a photon is isolated if the amount of hadronic transverse energy $E_{T}^{\text {had }}$, deposited inside a cone with aperture $R$ centered around the photon direction in the pseudo-rapidity and azimuthal angle plane, is smaller than some value $E_{T}^{\max }$. Both the $\mathrm{H} 1$ and ZEUS collaborations take $R \sim 1.0$ and $E_{T}^{\max } \sim 1 \mathrm{GeV}[1,2,3]$. 



Figure 2: Differential cross sections of the inclusive deep inelastic prompt photon production at HERA as a function of $E_{T}^{\gamma}, \eta^{\gamma}$, and $Q^{2}$ calculated at $4<E_{T}^{\gamma}<15 \mathrm{GeV},-0.7<\eta^{\gamma}<0.9,10<Q^{2}<350 \mathrm{GeV}^{2}, E_{e}^{\prime}>$ $10 \mathrm{GeV}, 139.8^{\circ}<\theta_{e}^{\prime}<171.9^{\circ}$ and $W_{X}>5 \mathrm{GeV}$. Notation of the histograms is as in Fig. 1 The experimental data are from ZEUS [2].


Figure 3: Differential cross sections of the deep inelastic prompt photon and jet associated production as a function of $E_{T}^{\gamma}, \eta^{\gamma}$ and $Q^{2}$ calculated at $3<E_{T}^{\gamma}<10 \mathrm{GeV}, E_{T}^{\text {jet }}>2.5 \mathrm{GeV},-1.2<\eta^{\gamma}<1.8,-1.0<\eta^{\text {jet }}<$ 2.1, $4<Q^{2}<150 \mathrm{GeV}^{2}, E_{e}{ }^{\prime}>10 \mathrm{GeV}, 153^{\circ}<\theta_{e}^{\prime}<177^{\circ}, y>0.05$ and $W_{X}>50 \mathrm{GeV}$. Notation of the histograms is as in Fig. 1. The experimental data are from H1 [3].

\section{Numerical results}

Our predictions on the differential cross sections of the deep inelastic inclusive prompt photon production as a function of transverse energy $E_{T}^{\gamma}$, pseudo-rapidity $\eta^{\gamma}$ and $Q^{2}$ variables are shown in Fig. 1 and Fig. 2 in comparison with the data [2,3]. We find that the predicted cross sections agree well with the $\mathrm{H} 1$ and ZEUS data both in the normalization and shape. A slight underestimation of the H1 data is only observed at high $Q^{2}$ (see [10]).

The results of our calculations for the jet-associated production of prompt photons are shown in Fig. 3 and Fig. 4 in comparison with the H1 [3] and ZEUS [1] data. One can see that the distributions measured by the $\mathrm{H} 1$ collaboration are well reproduced by our calculations. However, our results overeshoot the ealier ZEUS data. A possible reason for that can be connected with the jet selection algorithm being the consequence of the used approximations. Another possible reason is that our calculations are restricted to the parton level only, while the jet fragmentation mechanism could also be of importance.

\section{Conclusions}

We have studied both the inclusive and jet associated production of prompt photons at HERA 



Figure 4: Differential cross sections of the deep inelastic prompt photon and jet associated production as a function of $E_{T}^{\gamma}, \eta^{\gamma}$, and $E_{T}^{\text {jet }}$ calculated at $5<E_{T}^{\gamma}<10 \mathrm{GeV}, E_{T}^{\text {jet }}>6 \mathrm{GeV},-0.7<\eta^{\gamma}<0.9,-1.5<\eta^{\text {jet }}<$ $1.8, Q^{2}>35 \mathrm{GeV}^{2}, E_{e}^{\prime}>10 \mathrm{GeV}$ and $139.8^{\circ}<\theta_{e}^{\prime}<171.8^{\circ}$. Notation of the histograms is as in Fig.1. The experimental data are from ZEUS [1].

in the $k_{T}$-factorization approach. Our numerical predictions on the inclusive production cross sections are in good agreement with the H1 and ZEUS data. Our results for the jet associated production agree with the $\mathrm{H} 1$ measurements but overshoot the ZEUS data.

\section{Acknowledgements}

We are very grateful to DESY Directorate for the support in the framework of Moscow DESY project on Monte-Carlo implementation for HERA - LHC. Also this research was supported by the FASI of Russian Federation (grant NS-1456.2008.2), FASI state contract 02.740.11.0244 and RFBR grant 08-02-00896-a. N.Z. thanks Organizing Committee and ZEUS Collaboration for financial support.

\section{References}

[1] S. Chekanov et al. (ZEUS Collaboration), Phys. Lett. B 595, 86 (2004).

[2] S. Chekanov et al. (ZEUS Collaboration), DESY 09-142, arXiv:0909.4223 [hep-ex].

[3] F.D. Aaron et al. (H1 Collaboration), Eur. Phys. J. C 54, 371 (2008).

[4] A. Gehrmann-De Ridder, T. Gehrmann, and E. Poulsen, Phys. Rev. Lett. 96, 132006 (2006).

[5] D. Saxon, contribution to this Workshop (presented by T. Haas).

[6] A.V. Lipatov and N.P. Zotov, Phys. Rev. D 72, 054002 (2005).

[7] A.V. Lipatov and N.P. Zotov, J. Phys. G 34, 219 (2007); S.P. Baranov, A.V. Lipatov, and N.P. Zotov, Phys. Rev. D 77, 074024 (2008); Eur. Phys. J. C 56, 371 (2008).

[8] S. Chekanov et al. (ZEUS Collaboration), Eur. Phys. J. C 49, 511 (2007).

[9] F.D. Aaron et al. (H1 Collaboration), DESY 09-135, arXiv:0910.5631 [hep-ex].

[10] S.P. Baranov, A.V. Lipatov, and N.P. Zotov, Phys. Rev. D 81, 094034 (2010).

[11] M.A. Kimber, A.D. Martin, and M.G. Ryskin, Phys. Rev. D 63, 114027 (2001); G. Watt, A.D. Martin, and M.G. Ryskin, Eur. Phys. J. C 31, 73 (2003).

[12] A.D. Martin, W.J. Stirling, R.S. Thorne, and G. Watt, Eur. Phys. J. C 63, 189 (2009), arXiv:0901.0002 [hep-ph]. 